\title{
ACTIVIDAD ANTIBACTERIANA Y FUNGICIDA DE LAS ANTRAQUINONAS DE Aloe vera L. COMBINADAS CON CATIONES COBRE, HIERRO, PLATA Y BISMUTO
}

\author{
Antimicrobial and fungicide activity of anthraquinones from Aloe vera L. combined with copper, \\ iron, silver and bismuth cations
}

Félix E Saavedra ${ }^{1}$, Benedicta C López ${ }^{2}$, Victoria J Yrei ${ }^{2}$, Teresa C Gallardo², Neptalí Ale ${ }^{3}$ y Gloria C Gordillo ${ }^{4}$

${ }^{1}$ Instituto de Investigación en Ciencias Farmacéuticas y Recursos Naturales “Juan de Dios Guevara”. ${ }^{2}$ Centro Latinoamericano de Enseñanza e Investigación en Bacteriología Alimentaria - CLEIBA. ${ }^{3}$ Laboratorio Química Analítica e Instrumentación. Facultad de Química de Ing. Química. "Instituto de Química Biológica, Microbiología y Biotecnología "Marco Antonio Garrido Malo”

\section{RESUMEN}

El presente estudio tuvo por finalidad evaluar la actividad antibacteriana y fungicida de los macerados acuosos y alcohólicos de las hojas de Aloe vera L., que contenían derivados antraquinónicos. Con dichos macerados se prepararon

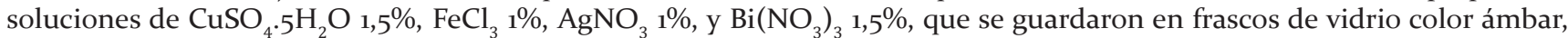
herméticamente cerrados por 12 meses, bajo refrigeración; un grupo similar se conservó a $25^{\circ} \mathrm{C}$. Se determinó la actividad de estos preparados, usando el método excavación placa de la USP XXX frente a cepas de Staphylococcus aureus, Escherichia coli, Pseudomonas aeruginosa y Aspergillus niger. Las soluciones acuosas de Aloe vera L. presentaron actividad antibacteriana en orden decreciente $\mathrm{Cu}>\mathrm{Ag}>\mathrm{Fe}$, y las soluciones alcohólicas $\mathrm{Ag}>\mathrm{Cu}>\mathrm{Fe}$. Solamente las soluciones acuosas de sulfato de cobre presentaron actividad fungicida. Los preparados conservados bajo refrigeración, presentaron mayor actividad antimicrobiana y fungicida que los preparados equivalentes conservados a $25^{\circ} \mathrm{C}$.

Palabras clave: Aloe vera L., antraquinonas, cationes cobre y plata, antimicrobiano, fungicida.

\section{SUMMARY}

This study aimed to evaluate the antimicrobial and fungicidal activity of aqueous and alcoholic macerated from leaves of Aloe vera L., containing anthraquinone derivatives. With this macerated liquids were prepared solutions of $\mathrm{CuSO}_{4} \cdot 5 \mathrm{H}_{2} \mathrm{O}$ $1,5 \%, \mathrm{FeCl}_{3} 1 \%, \mathrm{AgNO}_{3} 1 \%$, and $\mathrm{Bi}\left(\mathrm{NO}_{3}\right)_{3} 1,5 \%$, which were stored in amber glass flasks, hermetically closed for 12 months under refrigeration; a similar batch was kept at $25^{\circ} \mathrm{C}$. Antimicrobial and fungicide activities were determined on these preparations using plate excavation method, of USP XXX, against Staphylococcus aureus, Pseudomonas aeruginosa, Escherichia coli and Aspergillus niger strains. Aqueous solutions of Aloe vera $\mathrm{L}$. showed antimicrobial activity in decreasing order $\mathrm{Cu}>\mathrm{Ag}>\mathrm{Fe}$ and alcoholic solutions in also decreasing order $\mathrm{Ag}>\mathrm{Cu}>\mathrm{Fe}$. Only copper sulphate aqueous solutions showed fungicidal activity. The preparations preserved under refrigeration, had greater antimicrobial and fungicidal activity than equivalent preparations preserved at room temperature of $25^{\circ} \mathrm{C}$.

Keywords: Aloe vera L., anthraquinones, cooper and silver cations, antimicrobial fungicide.

\section{INTRODUCCIÓN}

a resistencia y multiresistencia de los microorganismos patógenos a los principales antibióticos es un tema que preocupa a las entidades de salud pública en todo el mundo, los reportes de 130 hospitales en Norteamérica, África, AsiaPacífico y Europa demostraron que, en 102829 cultivos de microorganismos patógenos, el $40 \%$ era resistente a la amoxicilina; mientras que para ampicilina oscilaba entre $53,96 \%$ y $71,22 \%$ en Norteamérica y Europa, respectivamente, sin considerar las infecciones en pacientes quemadoscausadas pormicroorganismos multirresistentes que se potencian por la automedicación ${ }^{(1)}$.
Una cepa de Staphylococcus aureus resistente a meticilina, descubierta en la década de los sesenta, ha desafiado a casi todos los antibióticos. De acuerdo al Laboratorio Central de Salud Pública de Londres, la bacteria puede infectar los pulmones, huesos y se puede diseminar vía sanguínea, siendo responsable de miles de muertes en el mundo ${ }^{(2)}$.

Por otro lado, el sulfato de cobre se utiliza como astringente en solución acuosa $0,25 \%$ para la conjuntivitis y lavados íntimos, y en solución acuosa $5 \%$ aplicada a tiempo, neutraliza las quemaduras originadas por el fósforo blanco formando fosfito de cobre insoluble ${ }^{(3)}$, esta sal se incluyó en el estudio de la detección y resistencia a 
antibióticos de Escherichia coli y Salmonella en aguas de regadío, demostrándose que de 20 cepas de Salmonella, 12 presentaron resistencia a tetraciclina y de 46 cepas de Escherichia coli analizadas, o9 fueron resistentes a la tetraciclina, 38 a estreptomicina y solo una a gentamicina. Todas las cepas de Salmonella tuvieron altos niveles de resistencia a $\mathrm{CuSO}_{4} \cdot 5 \mathrm{H}_{2} \mathrm{O}$ en dosis 1200 y $1600 \mu \mathrm{g} / \mathrm{mL}^{-1}$.

Asimismo, se ha determinado la concentración mínima inhibitoria (CMI) de $\mathrm{CuSO}_{4} \cdot 5 \mathrm{H}_{2} \mathrm{O}$ en cepas de Xanthomonas campestris, considerándose resistentes las que crecían a $200 \mu \mathrm{g} / \mathrm{mL}^{-1(4)}$. De otro lado, los iones plata y cobre, formados a partir de las sales $\mathrm{CuCl}_{2}$ y $\mathrm{AgCl}$, han demostrado actividad antibacteriana contra cepas de Pseudomonas aeruginosa, Stenotrophomonas maltophilia y Acinetobacter baumannii en sistemas de agua en hospitales ${ }^{(5)}$.

El uso de la sábila (Aloe vera L.) en medicina tradicional es muy difundido, la savia que fluye de las hojas recién cortadas-que contiene derivados antraquinónicos-, se deja secar y solidifica formando una masa marrónoscura brillante ( $28 \%$ de isobarbaloina anhidra) que se caracteriza por sus propiedades laxantes y purgantes ${ }^{(6,7)}$. El gel sin antraquinonas, se separa con espátula y se ingiere sólo o mezclado con jugos de frutas, para el tratamiento de afecciones de los sistemas respiratorio, digestivo, muscular, óseo y articular ${ }^{(8-13)}$. Para uso externo, se recomienda el gel fresco conteniendo antraquinonas, útil en el tratamiento de enfermedades de los ojos, piel, heridas externas e incluso en las quemaduras del holocausto de Hiroshima ${ }^{(14)}$. Por sus propiedades regeneradoras y bioestimulantes, el gel de sábila se utiliza en las industrias alimenticia, farmacéutica y cosmética ${ }^{(15)}$.

Las antraquinonas obtenidas por métodos cromatográficos, a partir de los extractos frescos o secos de las hojas de diferentes variedades de Aloe (Aloe vera L., Aloe barbadensisy Aloe ferox han demostrado propiedades antibacterianas, fungicidas y antivirales ${ }^{(16-19)}$.

Además del sulfato de cobre pentahidratado $\left(\mathrm{CuSO}_{4} \cdot 5 \mathrm{H}_{2} \mathrm{O}\right)$, este ensayo también motivó el empleo de sales inorgánicas como nitrato de plata $\mathrm{AgNO}_{3}$, utilizado desde hace mucho tiempo por su propiedad astringente, en soluciones diluidas, cauterizante y bactericida, en soluciones del 2 al 5\%; cloruro férrico $\mathrm{FeCl}_{3}$, utilizado para la cuantificación de los derivados antraquinónicos de Aloe vera ${ }^{(20)}$; y nitrato de bismuto $\mathrm{Bi}\left(\mathrm{NO}_{3}\right)_{3}$, prescrito junto con omeprazol, amoxicilina y metronidazol para el tratamiento de la úlcera péptica ${ }^{(21)}$.

El objetivo del presente trabajo fue investigar si las sales mencionadas, combinadas con derivados antraquinónicosdelos maceradosacuososyalcohólicos de Aloe vera L., poseen actividad antibacteriana y fungicida frente a Staphylococcus aureus, Escherichia coli, Pseudomonas aeruginosa y Aspergillus niger.

\section{MATERIALES Y MÉTODOS}

\section{Cepas microbianas}

Los microorganismos de ensayo seleccionados para estudiar la actividad bactericida fueron dos especies representativas de bacterias Gram negativas: Escherichia coli ATCC 8739 y Pseudomonas aeruginosa ATCC 27953, proporcionadas por el Instituto de Medicina Tropical, UNMSM; y una especie representativa Gram positiva: Staphylococcus aureus ATCC 29737. Para la actividad antifúngica se utilizó la cepa del hongo Aspergillus niger ATCC 16404.

\section{Macerados acuosos y alcohólicos de Aloe vera L}

La preparación de los macerados acuosos y alcohólicos de Aloe vera L., que contenían derivados antraquinónicos, sales $\mathrm{CuSO}_{4} \cdot \mathrm{H}_{2} \mathrm{O} \quad 1,5 \%, \mathrm{FeCl}_{3} 1 \%$, $\mathrm{AgNO}_{3} 1 \%, \mathrm{Bi}\left(\mathrm{NO}_{3}\right)_{3} 1,5 \%$, y su conservación en refrigeración y a $25^{\circ} \mathrm{C}$, se hizo según procedimientos descritos por Saavedra y col. ${ }^{(23)}$.

\section{Actividad antibacteriana}

Para la evaluación de la actividad antibacteriana y fungicida delasdiferentessolucionesseutilizóla técnicadeexcavaciónplaca-agar, según método de la USP $30 / \mathrm{NF}_{2} 5^{(23)}$.

Ensayos en placa con $\mathrm{AgNO}_{3}$ o, o1 M y $\mathrm{CuSO}_{4} \cdot 5 \mathrm{H}_{2} \mathrm{O}$ o.o1M, al o, 5; 1, o; 1,5; 2 ; 3 y $5 \%$

Serealizaroncon la finalidad deevaluarla concentración mínima de actividad antibacteriana y comparar dicha actividad cuando estas sales se combinan con los macerados acuosos y alcohólicos de Aloe vera $\mathrm{L}$.

\section{RESULTADOS}

Experimentalmente, quedó establecido que, tanto los macerados acuosos como alcohólicos, de hojas de Aloe vera L., en ambos casos líquidos marrón-rojizo (sin sales inorgánicas), y conservados bajo refrigeración por uno, ocho, diez, doce y veinticuatro meses, carecen de actividad germicida y fungicida.

El sulfato de cobre y nitrato de plata, combinados con los macerados acuosos y alcohólicos de Aloe vera L., demostraron actividad antibacteriana y, solamente la sal de cobre, presentó actividad fungicida.

Por este motivo, para comparar, se determinó la actividad antibacteriana de soluciones acuosas diluidas de estas sales, resultando que el sulfato de cobre o,o1M 
al $0,25 \%$ carece de actividad antibacteriana y fungicida, mientras que del $1,5 \%$ al $5 \%$, es bactericida.

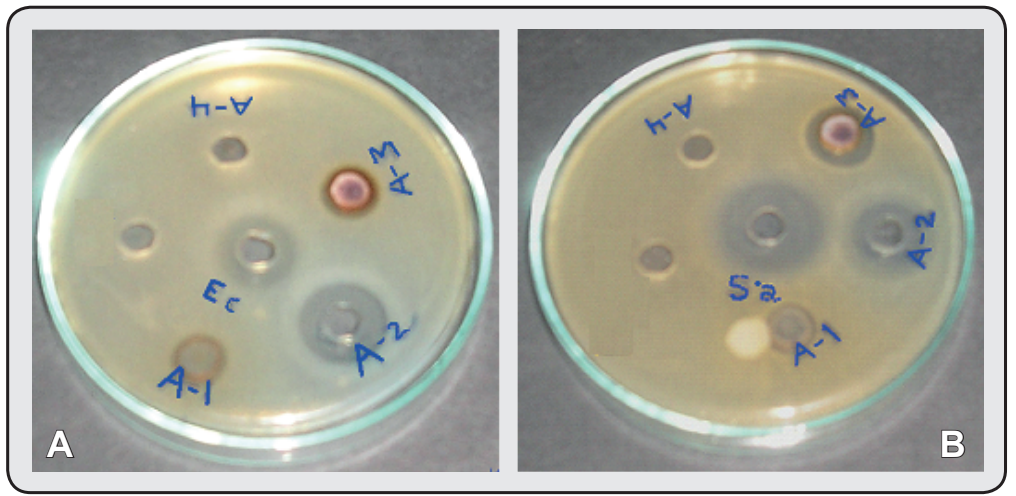

Figura 1.Efecto del macerado acuoso de hojas de Aloe vera L con derivados antraquinónicos y adición de salesinorgánicas conservados a $25^{\circ} \mathrm{C}$ A) Ensayo con E. coli B) Ensayo con $S$. aureus.

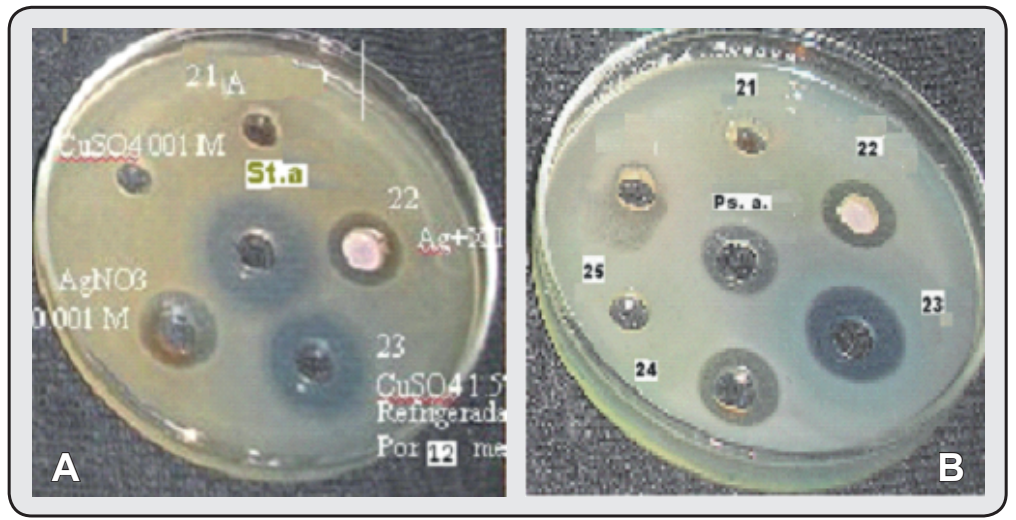

Figura 2. Efecto del macerado acuoso de hojas de Aloe vera L con derivados antraquinónicos y adición de sales inorgánicas conservados bajo refrigeración A) Ensayo con $S$. aureus. B) Ensayo con Pseudomonas aeruginosa. Efecto de sales diluidas.
En la tabla № 1 y figuras 1 y 2, se presentan los resultados de los ensayos de actividad antibacteriana y fungicida de los macerados con adición de sales inorgánicas, conservados en frascos ámbar herméticamente cerrados por doce meses, tanto a $25^{\circ} \mathrm{C}$ como bajo refrigeración. También se presenta el efecto de soluciones de $\mathrm{CuSO}_{4}$ y $\mathrm{AgNO}_{3}$ o,oiM.

Sedemuestra que tanto el combinado de Aloe vera L. con bismuto, conservado a $25^{\circ} \mathrm{C}$, como el puesto en refrigeración por doce meses, no presentan actividad antibacteriana ni fungicida frente a las cepas estudiadas.

Los preparados acuosos con $\mathrm{FeCl}_{3}$ conservados a $25^{\circ} \mathrm{C}$ y bajo refrigeración, presentan baja actividad antibacteriana $\mathrm{y}$ ausencia de efecto fungicida frente a Aspergillus niger.

Los preparados acuosos con plata $1 \%$, tienen efecto inhibitorio de crecimiento bacteriano menor respecto a los de cobre $1,5 \%$. Asimismo, las dos soluciones acuosas de $\mathrm{CuSO}_{4} 5 \mathrm{H}_{2} \mathrm{O}$, conservadas a $25^{\circ} \mathrm{C}$ y en refrigeración, muestran inhibición del crecimiento de Aspergillus niger, lo que no se observa con el preparado de nitrato de plata. Respecto a las sales de cobre y plata, en la fig. 2 se aprecia la actividad antibacteriana de la solución acuosa de $\mathrm{AgNO}_{3}$ o,olM frente a $S$. aureus y ausencia de la misma con $\mathrm{CuSO}_{4} \cdot 5 \mathrm{H}_{2} \mathrm{O}$ o,olM.

En la fig. 2, se observa que frente a $S$. Aureus los halos de inhibición del sulfato de cobre $1,5 \%$, combinado con el macerado acuoso de Aloe vera L. y refrigerado, son similares a los halos de inhibición de los ensayos con soluciones acuosas recientes de sulfato de cobre $5 \%$ que se muestran en la Fig. 3 .

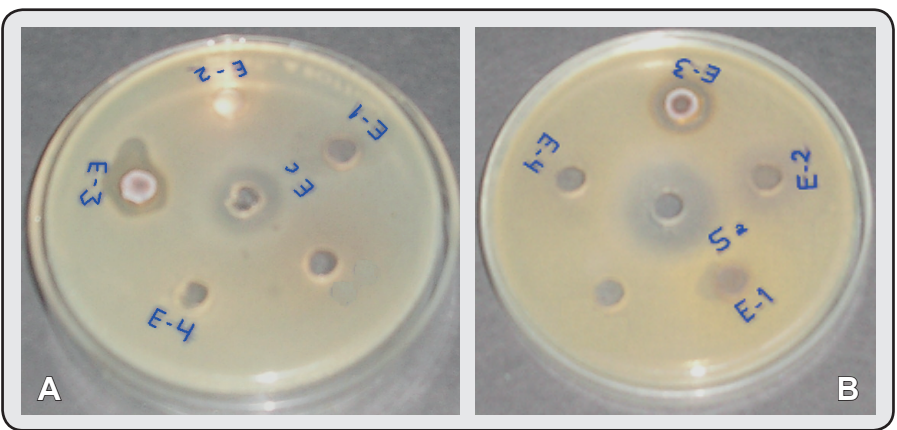

Figura 4. Efecto del macerado alcohólico de Aloe vera L. con derivados antraquinónicos y adición de sales inorgánicas conservado a $25^{\circ} \mathrm{C}$ A) Ensayo con Escherichia coli. B) Ensayo con Staphylococcus aureus. 


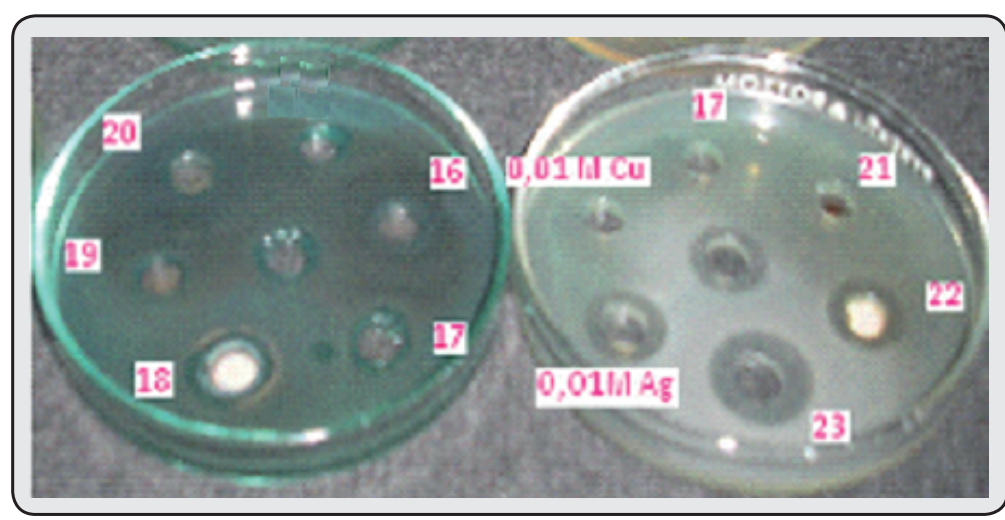

Figura 5. Ensayos con Pseudomonas aeruginosa y preparados conservados bajo refrigeración por doce meses. Efecto de sales inorgánicas.

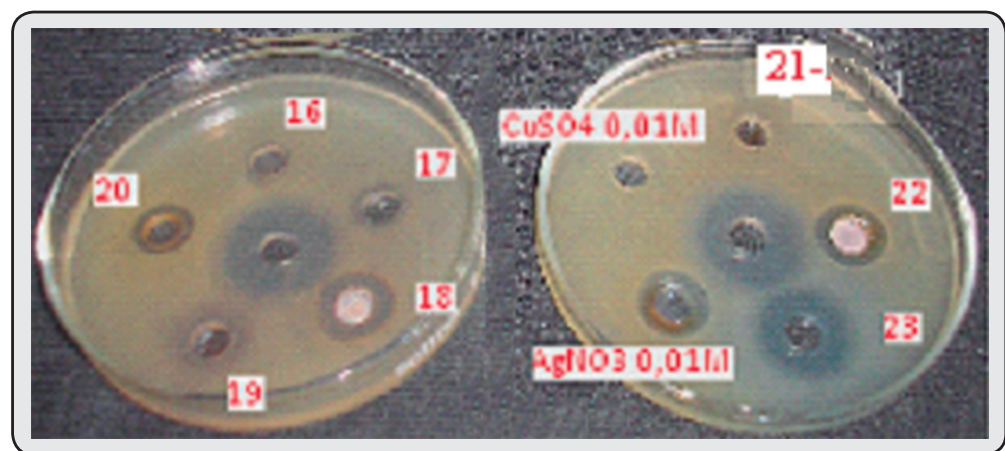

Figura 6. Ensayos con Pseudomonas aeruginosa y preparados conservados bajo refrigeración por doce meses. Efecto de sales inorgánicas.

En la tabla № 2 y figura 4, se observa que de los ocho macerados alcohólicos con sales inorgánicas, dos para cada sal, destacan los dos preparados con nitrato de plata y uno con bismuto, por presentar bajo efecto inhibitorio en el crecimiento de las cepas estudiadas, excepto Aspergillus niger. Las demás soluciones carecen de efecto antibacteriano y fungicida.

Las figuras 5 y 6 comparan la actividad antibacteriana de algunos de los preparados investigados frente a Pseudomonas aeruginosa y Staphylococcus aureus, destacando los de sales de cobre y plata, a excepción de la solución de $\mathrm{CuSO}_{4}{ }_{5} \mathrm{H}_{2} \mathrm{O}$ o,o1 M, equivalente a o, $25 \%$, que no presentó actividad.

La figura 7 presenta los halos de inhibición del crecimiento de $A$. niger incubado por siete días. Se destaca el efecto inhibitorio residual del crecimiento del microorganismo con el preparadoacuoso de sulfato decobre (A-2), conservado a temperatura ambiente por doce meses. Las demás muestras corresponden al estudio realizado.

\section{DISCUSIÓN}

En el estudio se destacó la actividad antibacteriana y fungicida del sulfato de cobre combinado con los macerados acuosos de Aloe vera L., conteniendo heterósidos antraquinónicos, lo que permite recordar que las antraciclinas daunorrubicina y doxorrubicina o adriamicina -antibióticos antineoplásicostienen estructura antraquinónica enlazada con un aminoazúcar, y para explicar el mecanismo de acción de estos antibióticos, se considera que el encaje en la cadena del ADN, se debe a la afinidad del grupo amino de estos antibióticos por los fosfatos del ADN, ocasionando la inestabilidad de este complejo y obstaculizando la replicación del mismo, la trascripción y la traducción, con el consecuente daño que conduce a la muerte de la célula tumoral ${ }^{(24)}$. De manera análoga, puede proponerse que, los azúcares (glucosa, manosa, xilosa) de los aglicones unidos a los derivados antracénicos de laaas diferentes variedades de Aloe y otras especies vegetales con actividad laxante como sen, ruibarbo, cáscara sagrada, frángula, participan o son necesarios, para lograr la estereoquímica deseada y puedan desarrollar su efecto antibacteriano y fungicida, reforzando esta hipótesis la formación del quelato de cobre descrito en estudios previos ${ }^{(22)}$.

El incremento de actividad antibacteriana en algunos de los preparados conservados bajo refrigeración puede explicarse con la teoría de Filatov, quien postulaba que la actividad bioestimulante de Aloe vera L. se incrementa cuando las hojas de esta planta se conservan en condiciones adversas de

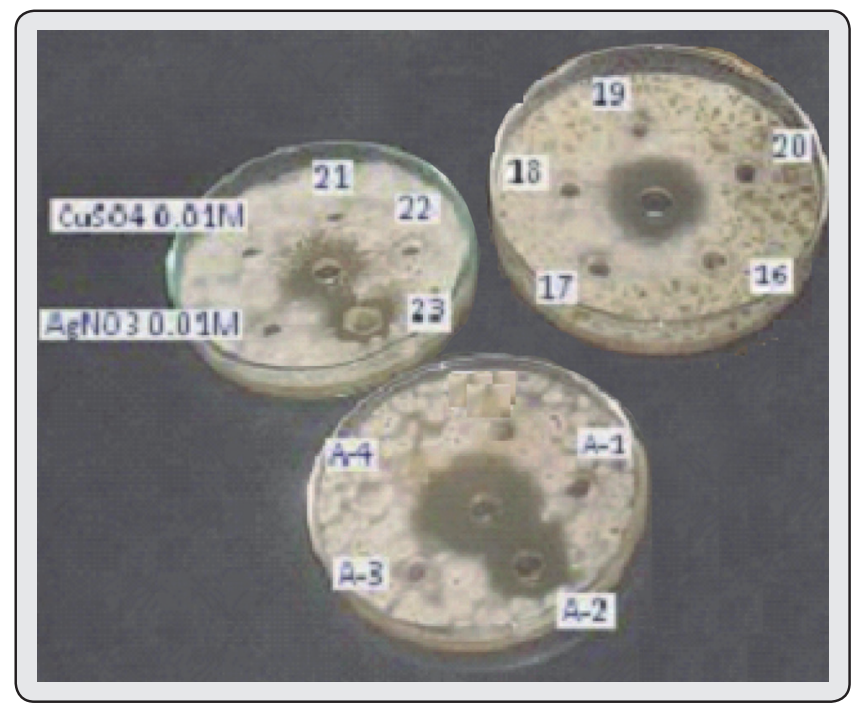

Figura 7. Ensayos con Aspergillus niger, vista después de una semana. 
Tabla 1. Actividad antimicrobiana y fungicida de macerados acuosos de hojas de Aloe vera L. con y sin adición de sales inorgánicas; soluciones conservadas por doce meses a $25^{\circ} \mathrm{C}$ y bajo refrigeración.

\begin{tabular}{|c|c|c|c|c|c|c|c|c|c|}
\hline Condición & Código & S.a. & Oxit. & E.c. & Oxit. & Ps. a. & Oxit. & Asp. $n$. & Nist. \\
\hline \multicolumn{10}{|l|}{$25^{\circ} \mathrm{C}$} \\
\hline Macerado con $\mathrm{FeCl}_{3} 1 \%$ & A-1 & 11 & 25 & 12 & 14 & - & 15 & & \\
\hline Macerado con $\mathrm{CuSO}_{4} \cdot 5 \mathrm{H}_{2} \mathrm{O} 1,5 \%$ & A-2 & $16,5(*)$ & $22,5(*)$ & $18(*)$ & $16,5(*)$ & $14,5(*)$ & $13,5(*)$ & $20(*)$ & $23,5(*)$ \\
\hline Macerado con $\mathrm{AgNO}_{3} 1 \%$ & A-3 & $16\left(^{*}\right)$ & $22,5(*)$ & $13(*)$ & $6,5\left(^{*}\right)$ & 16 & 13 & - & 17 \\
\hline $\begin{array}{c}\text { Macerado con } \mathrm{Bi}\left(\mathrm{NO}_{3}\right)_{3} 1,5 \% \\
\text { Refrigeradas }\end{array}$ & A- 4 & - & 25 & - & 14 & - & 15 & - & - \\
\hline Macerado con $\mathrm{FeCl}_{3} 1 \%$ & 20 & 13 & 23 & 12 & 20 & 13 & 13 & - & 26 \\
\hline Macerado con $\mathrm{Bi}\left(\mathrm{NO}_{3}\right)_{3} 1,5 \%$ & 21 & - & 22 & - & 18 & - & 15 & - & 25 \\
\hline Macerado con $\mathrm{AgNO}_{3} 1 \%$ & 22 & 15 & 22 & 14 & 18 & 15 & 15 & - & 25 \\
\hline $\begin{array}{c}\text { Macerado con } \mathrm{CuSO}_{4} \cdot 5 \mathrm{H}_{2} \mathrm{O} 1,5 \% \\
\text { Sólo sales inorgánicas }\end{array}$ & 23 & 19 & 22 & 19 & 18 & 18 & 15 & 25 & 25 \\
\hline $\mathrm{AgNO}_{3} 0,01 \mathrm{M}$ & 24 & 16 & 22 & 15 & 18 & 16 & 15 & - & 25 \\
\hline $\mathrm{CuSO}_{4} 0,01 \mathrm{M}$ & 25 & - & 22 & - & 18 & - & 15 & - & 25 \\
\hline
\end{tabular}

$\left.{ }^{\star}\right)$ Promedio en $\mathrm{mm}$ de zona de inhibición

iluminación y temperatura ${ }^{(25)}$. Esta teoría permite sugerir la participación de sustancias solubles en agua provenientes del gel de sábila -además de los derivados antraquinónicos- las cuales al difundir en el medio de cultivo, junto con las moléculas de las sales inorgánicas, presentan mayor actividad antibacteriana y fungicida que los preparados equivalentes conservados a $25^{\circ} \mathrm{C}$.

Por otro lado, el halo inhibitorio de crecimiento bacteriano, correspondiente a la solución acuosa $1,5 \%$ de sulfato de cobre, es de menor diámetro - casi equivalente a $1 / 4$ del halo obtenido - cuando se utiliza la misma concentración de la sal de cobre y como solvente los macerados acuosos de las hojas de Aloe vera $\mathrm{L}$. Esto refuerza la hipótesis de la participación de sustancias hidrosolubles del gel de sábila presentes en los macerados acuosos. Asimismo, la interacción entre el macerado acuoso de sábila y la sal de cobre, origina un residuo cristalino de color rojo oscuro correspondiente a óxido cuproso $\mathrm{Cu}_{2} \mathrm{O}$, es decir, la reducción del cobre implica la formación de quelato de cobre ${ }^{(23)}$, el cual sería responsable de la potenciación de actividad antibacteriana del catión cobre.
De manera comparativa, los cuatro macerados alcohólicos con sales inorgánicas conservados bajo refrigeración, presentan pequeña actividad antibacteriana, respecto a los preparados equivalentes conservados a $25^{\circ} \mathrm{C}$, incluyendo al de la sal de cobre a pesar de su escasa solubilidad en alcohol.

\section{CONCLUSIONES}

a. Los macerados acuosos y alcohólicos de hojas de Aloe vera L sin adición de sales inorgánicas, refrigerados por diferentes periodos carecen de actividad antibacteriana y fungicida frente a las cepas Staphylococcus aureus, Escherichia coli, Pseudomonas aeruginosa y Aspergillus niger.

b. Los macerados acuosos de hojas de Aloe vera L. con $\mathrm{FeCl}_{3} 1 \%$ y $\mathrm{Bi}\left(\mathrm{NO}_{3}\right)_{3} 1,5 \%$, almacenados a temperatura ambiente $\mathrm{y}$ bajo refrigeración presentan cierta actividad antibacteriana y carecen de actividad fungicida.

c. El maceradoacuoso de hojas de Aloevera L. con $\mathrm{CuSO}_{4}$ $1,5 \%$, conservado bajo refrigeración por doce meses,

Tabla 2. Actividad antibacteriana y fungicida de macerados alcohólicos de hojas de Aloe vera L con derivados antraquinónicos y adición de sales inorgánicas; soluciones guardadas doce meses a $25^{\circ} \mathrm{C}$ y bajo refrigeración.

\begin{tabular}{|c|c|c|c|c|c|c|c|c|c|}
\hline $25^{\circ} \mathrm{C}$ & Código & S.a. & Oxit. & E.c. & Oxit. & Ps. a. & Oxit. & Asp. $\mathrm{n}$. & Nist. \\
\hline Macerado con $\mathrm{FeCl}_{3} 1 \%$ & E-1 & - & 20 & - & 15 & & & & \\
\hline Macerado con $\mathrm{CuSO}_{4} 1,5 \%$ & $\mathrm{E}-2$ & - & 20 & - & 15 & & & & \\
\hline Macerado con $\mathrm{AgNO}_{3} 1 \%$ & E-3 & $15\left(^{*}\right)$ & $19,5\left(^{*}\right)$ & $17\left(^{*}\right)$ & $17\left(^{*}\right)$ & 20 & 14 & - & 17 \\
\hline $\begin{array}{l}\text { Macerado con } \mathrm{Bi}\left(\mathrm{NO}_{3}\right)_{3} 1,5 \% \\
\text { Refrigeradas }\end{array}$ & E-4 & - & 20 & - & 15 & & & & \\
\hline Macerado con $\mathrm{FeCl}_{3} 1 \%$ & 16 & 10 & 23 & 10 & 20 & 10 & 12 & - & 26 \\
\hline Macerado con $\mathrm{Bi}\left(\mathrm{NO}_{3}\right)_{3} 1,5 \%$ & 17 & 11 & 23 & - & 20 & $11,5(*)$ & $14(*)$ & - & 26 \\
\hline Macerado con $\mathrm{AgNO}_{3} 1 \%$ & 18 & 16 & 23 & 15 & 20 & 17 & 13 & - & 26 \\
\hline Macerado con $\mathrm{CuSO}_{4}$ 1,5\% & 19 & 13 & 23 & 11 & 20 & 10 & 13 & - & 26 \\
\hline
\end{tabular}

$\left.{ }^{*}\right)$ Promedio en mm de zona de inhibición 
presenta mayor actividad antibacteriana y fungicida que el preparado equivalente conservado a $25^{\circ} \mathrm{C}$.

d. Los preparados alcohólicos de hierro, cobrey bismuto almacenados bajo refrigeración presentan bajo efecto antimicrobiano y carecen de actividad fungicida.

Agradecimientos.- Al Dr. Fernando Quevedo Ganoza, Director del CLEIBA de la Facultad de Farmacia y B.Q.UNMSM, a los Doctores Luis Solano Mendoza y Jorge Chumpitaz Conde del Instituto de Medicina TropicalUNMSM, a la Dra. Elba Lau, Gerente de Control de Calidad de Laboratorios MEDIFARMA y al Mg. José Apesteguía Infante por su valiosa colaboración.

\section{REFERENCIAS BIBLIOGRÁFICAS}

1. Ortiz F, Morales I, Gil A, Reyna J, Benítez A, Aldrete J y col. El reto de la resistencia bacteriana en México: los beneficios de contar con una nueva alternativa de manejo antimicrobiano eficaz. Med Int Mex 2009; 25(5):361-71.

2. BioHealth Partnership. Disminución de tasas de Infección en Hospitales e Institutos de Salud: el rol de las aleaciones de cobre en el combate contra los organismos infecciosos. $2^{\mathrm{a}}$ ed. BioHealth Partnership. 2009. p. 12-18.

3. Belikov VG. Farmashevticheskaya Ximia. Vuishaya Shkola. Moscú, 1985. p. 253-7.

4. López O, León J, Jiménez M, Chaidez C. Detección y resistencia a antibióticos de Escherichia coliy Salmonella en agua y suelo agrícola. Rev Fitotec Mex 2009; 32(2): 119-26.

5. Huang HI, Shih HY, Lee CM, Yang TC, Lay JJ, Lin YE. In Vitro efficacy of copper and silver ions in eradicating Pseudomonas aeruginosa, Stenotrophomponasmaltophiliaand Acinetobacter baumannii: Implications for on-site disinfection for hospital infection control. Water Res 2008; 42(1-2): 73-80

6. Real Farmacopea Española. Ministerio de Sanidad y Consumo. Madrid, 1997. Tomo I, p. 396-8.

7. British Pharmacopoeia. Department of Health. Scottish Office - Crown Copyright. 1999. V.1, p. 63-5.

8. Rodríguez M, Hernandez M, Arias A, López R y Martínez Y. Acción antiasmática del Aloe vera en pacientes. Rev Cubana Plant Med 2004; 9(1). [En línea] Acceso 04 noviembre 2012. Disponible en: http://scielo.sld.cu/scielo. php?script=sci_arttext\&pid=S1028-47962004000100010

9. Visuthikosol V, Chowchuen B, Sukwanarat Y, Sriurairatana S, Boonpucknavig V. Effect of aloe vera gel to healing of burn wound a clinical and histologic study. J Med Assoc Thai 1995; 78(8): 403-9.

10. Lee J.K, Lee MK, Yun YP, Kim Y, Kim JS, Kim K, et al. Acemannam purified from Aloe vera induces phenotypic and functional maturation of immature dendritic cells. Int Immunopharmacol 2001; 1(7): 1275-84.
11. Borrelli F, Izzo A. The plant kingdom as a source antiulcer remedies. Phytother Res 2000; 14(8): 581-91.

12. Reynolds T. Dweck A C. Aloe vera leaf gel: a review update. J Ethnopharmacol 1999; 68(1-3): 3-37.

13. Gibaja S. Pigmentos naturales quinonicos. Fondo Edit. UNMSM. Lima, 1998. p. 75, 96-9.

14. Zawahry ME, Hegazy MR, Helal M. Use of aloe in treating leg ulcers and dermatoses. Int J Dermatol 1973; 12(1):68-73.

15. Eshun K, He Q. Aloe vera: A Valuable Ingredient for the Food, Pharmaceutical and Cosmetic Industries - A Review. Crit Rev Food Sci Nutr 2004; 44(2): 91-6.

16. Soeda M, Otomo M, Ome M, Kawashima K. Studies on antibacterial and anti-fungal activities of Cape aloe. Nihon Saikingaku Zasshi 1966; 21(10): 609-14.

17. Sydiskis RJ, Owen DG, Lohr JL, Rosler KH, Blomster RN. Inactivation of enveloped viruses by anthraquinones extracted from plants. Antimicrob Agents Chemother 1991; 35(12): 2463-6.

18. Rojas L, Matamoros M, Garrido N, Finlay C. Acción de un extracto acuoso de Aloe barbadensis Miller en cultivo in vitro de Trichomonas vaginalis. Rev Cubana Med Trop 1995; 47(3): 181-4.

19. Ali MI, Shalaby NM, Elgamal MH, Mousa AS. Antifungal effects of different plant extracts and their major components of selected aloe species. Phytother Res 1999; 13(5): 401-7.

20. Bustamante J, Carrascal L. Estandarización de la técnica espectrofotométrica (UV-VIS) para la cuantificación de antraquinonas presentes en productos a base de Aloe vera. Trabajo de Grado. Universidad Tecnológica de PereiraColombia. 2010. p. 39-41 (versión digital). [En línea] Acceso 04 noviembre 2012. Disponible en: http://repositorio.utp.edu.co/ dspace/bitstream/11059/1800/1/54308520218B982.pdf

21. Saavedra F, Ale N, Gordillo G, Apesteguía A, Jurado B, Revilla A. Análisis por espectroscopia UV y FTIR de macerados acuosos y alcohólicos de Aloe vera L. y Aloe barbadensis Miller. Interacción con sales inorgánicas. Rev Soc Quím Perú 2010; 76(3): 243-6o.

22. USP XXX/NF XXV Pharmacopoeia.

23. Rodilla F, Boletin Oncológico del área sanitaria de Teruel (España) $2000 \mathrm{~N}^{\circ} 15, \mathrm{vol} 3$.

24. Mashkowsky MD, Lekarstvenni Sriedsva (Sustancias medicinales). Medicina-Moscú. Moscú, 1967. T. 1, p. 208, 653-4.

Manuscrito recibido el: 10/09/2012

Aceptado para su publicación el: 10/12/2012

\section{Correspondencia:}

Nombre: Felix Saavedra Nizama

Dirección: Jr. Puno 1002 - Lima o1, Perú

e-mail: fesn2709@gmail.com 